\title{
Are there lessons from the cluster of neonatal deaths in Victoria for emergency physicians?
}

In October 2015 it became public knowledge that between January 2013 and August 2014 a cluster of ten neonatal deaths had occurred at an urban fringe hospital in Victoria. Of the ten deaths, an expert review concluded that seven were potentially avoidable. Understandably there were a number of reviews to try to find out what went wrong. They identified failures at multiple levels: clinical practice, clinical governance and regulatory bodies (including the Australian Health Practitioner Regulation Agency, the Australia Council on Healthcare Standards (regarding accreditation) and the Victorian Department of Health and Human Services).

At the clinical practice level, there were deficits in skills and training. At the governance level, there was a lack of appropriate clinical risk management including a lack of an appropriate mortality and morbidity review processes (at both unit and health service levels). At the regulatory level, there was slow and/or inadequate response to issues raised by clinicians about clinical risk and a failure of the accreditation process to identify important gaps in quality and safety. Importantly, rapid population growth and 'drift' in scope-of-practice (treating higher complexity patients) due in part to increasing patient numbers and community expectations were identified as significant contributing factors.

At first glance it might seem that these events are quite remote from emergency medicine practice, but look closer. Is Emergency Medicine's house in order with respect to clinical risk management? Emergency Departments are experiencing increases in attendances, some of which are very dramatic. Is the associated clinical risk being actively managed? Are larger emergency departments supporting smaller ones and local urgent care centres to identify and manage clinical risk?

Managing clinical risk in emergency departments should be an active process occurring at clinician, department, health service and, I would argue, regional levels. At the clinician level, all clinicians working in emergency services must have knowledge and skills to do this competently. How sure are we that this is the case? A particular challenge is maintaining the knowledge and skills of older clinicians or those working in smaller health services where training opportunities are limited. While there is regular work-based assessment and feedback during specialist training, formal structures for this after Fellowship are rare. Is there a case for more formal assessments of knowledge and skills through professional life? Are there clever ways to achieve this, perhaps using educational networks and technology?

At the departmental governance level, identification of risk is traditionally multimodal - complaints from patients, feedback from other departments, 'near miss' cases and mortality and morbidity reviews. While very useful, these are all reactive. Emergency departments can be proactive by undertaking regular scope-of-service reviews; particularly important if major changes in presentation numbers or case-mix occur. These focus on whether current models of care, processes and resources are appropriate in the face of increased demand. This approach is also useful if there are significant changes to the 'back end' of health serviced in terms of health service configuration or admission policies or high levels of access block. For example, with increased patient numbers

This is the author manuscript accepted for publication and has undergone full peer review but has not been through the copyediting, typesetting, pagination and proofreading process, which may lead to differences between this version and the Version of Record. Please cite this article as doi: $10.1111 / 1742-6723.12561$

This article is protected by copyright. All rights reserved. 
and high access block it can be easy for there to be a 'drift' towards admitting high risk patients to observation unit beds in order to meet the four hour target. A scope of practice review defining clear clinical parameters for patients unsuitable for observation unit care can mitigate this risk.

Clinicians must also speak out when they identify serious clinical risk, for example by notifying health services or regulatory authorities. It is not good enough to take an 'it'll be all right' attitude. Having an open and responsive, no-blame culture regarding safety and clinical risk is powerful for early identification of risk and prevention of adverse events. How open is the culture is your ED?

Mortality and morbidity (M\&M) reviews are also an important tool. A wide net of potential cases is likely to yield the most useful data. As well as ED deaths and ED-related morbidity, consider including unexpected deaths or ICU transfers within 24 hours of the index ED visit and for small services, high risk acute inter-hospital transfers and their outcome. M\&M meetings are usually internal. This runs the risk of 'forgiving' the foibles of an ED or health service without rigorous questioning. Examples of such foibles could include delays to responses by on-call specialists out of hours or unavailability of some investigational services over weekends. In my opinion, there are compelling arguments for M\&M meetings to be inter-health service, ideally on a regional basis. This allows some independence in the review process, facilitates cross-institutional learning from problems identified, encourages communication and shared processes for patients transferred between facilities and provides a mechanism for smaller emergency departments or urgent care centres to have their cases analysed and discussed.

At the health service involved here, the system failed its most vulnerable patients and at multiple levels. Clinical governance systems at all levels need to be stronger and more sophisticated. Emergency Medicine has a lot to learn from these events, but will we heed the lessons?

This article is protected by copyright. All rights reserved. 


\section{University Library}

\section{- M M N E R VA A gateway to Melbourne's research publications}

Minerva Access is the Institutional Repository of The University of Melbourne

Author/s:

Kelly, A-M

Title:

Are there lessons from the cluster of neonatal deaths in Victoria for emergency physicians?

Date:

2016-06-01

Citation:

Kelly, A. -M. (2016). Are there lessons from the cluster of neonatal deaths in Victoria for emergency physicians?. EMERGENCY MEDICINE AUSTRALASIA, 28 (3), pp.353-354. https://doi.org/10.1111/1742-6723.12561.

Persistent Link:

http://hdl.handle.net/11343/291033 